\title{
Interval Oscillation Criteria for Second-Order Dynamic Equations with Nonlinearities Given by Riemann-Stieltjes Integrals
}

\author{
Yuangong Sun \\ School of Mathematics, University of Jinan, Jinan, Shandong 250022, China \\ Correspondence should be addressed to Yuangong Sun, sunyuangong@yahoo.cn
}

Received 18 May 2011; Accepted 30 July 2011

Academic Editor: Paul Eloe

Copyright (C) 2011 Yuangong Sun. This is an open access article distributed under the Creative Commons Attribution License, which permits unrestricted use, distribution, and reproduction in any medium, provided the original work is properly cited.

By using a generalized arithmetic-geometric mean inequality on time scales, we study the forced oscillation of second-order dynamic equations with nonlinearities given by Riemann-Stieltjes integrals of the form $\left[p(t) \phi_{\alpha}\left(x^{\Delta}(t)\right)\right]^{\Delta}+q(t) \phi_{\alpha}(x(\tau(t)))+\int_{a}^{\sigma(b)} r(t, s) \phi_{\gamma(s)}(x(g(t, s))) \Delta \xi(s)=e(t)$, where $t \in\left[t_{0}, \infty\right)_{\mathbb{T}}=\left[t_{0}, \infty\right) \cap \mathbb{T}, \mathbb{T}$ is a time scale which is unbounded from above; $\phi_{*}(u)=$ $|u|^{*} \operatorname{sgn} u ; \gamma:[a, b]_{\mathbb{T}_{1}} \rightarrow \mathbb{R}$ is a strictly increasing right-dense continuous function; $p, q, e$ : $\left[t_{0}, \infty\right)_{\mathbb{T}} \rightarrow \mathbb{R}, r:\left[t_{0}, \infty\right)_{\mathbb{T}} \times[a, b]_{\mathbb{T}_{1}} \rightarrow \mathbb{R}, \tau:\left[t_{0}, \infty\right)_{\mathbb{T}} \rightarrow\left[t_{0}, \infty\right)_{\mathbb{T}}$, and $g:\left[t_{0}, \infty\right)_{\mathbb{T}} \times[a, b]_{\mathbb{T}_{1}} \rightarrow$ $\left[t_{0}, \infty\right)_{\mathbb{T}}$ are right-dense continuous functions; $\xi:[a, b]_{\mathbb{T}_{1}} \rightarrow \mathbb{R}$ is strictly increasing. Some interval oscillation criteria are established in both the cases of delayed and advanced arguments. As a special case, the work in this paper unifies and improves many existing results in the literature for equations with a finite number of nonlinear terms.

\section{Introduction}

Following Hilger's landmark paper [1], there have been plenty of references focused on the theory of time scales in order to unify continuous and discrete analysis, where a time scale is an arbitrary nonempty closed subset of the reals, and the cases when this time scale is equal to the reals or to the integers represent the classical theories of differential and of difference equations. The oscillation theory has been developed very rapidly since the discovery of time scale calculus with this understanding. Throughout this paper, a knowledge and understanding of time scale calculus is assumed. For an introduction to time scale calculus and dynamic equations, we refer to the seminal books by Bohner and Peterson [2,3]. 
In this paper, we consider the following second-order dynamic equation with the nonlinearity given by a Riemann-Stieltjes integral of the form

$$
\left[p(t) \phi_{\alpha}\left(x^{\Delta}(t)\right)\right]^{\Delta}+q(t) \phi_{\alpha}(x(\tau(t)))+\int_{a}^{\sigma(b)} r(t, s) \phi_{\gamma(s)}(x(g(t, s))) \Delta \xi(s)=e(t),
$$

where $t \in\left[t_{0}, \infty\right)_{\mathbb{T}}=\left[t_{0}, \infty\right) \cap \mathbb{T}, t_{0} \in \mathbb{T}, \mathbb{T}$ is a time scale (a closed nonempty subset of real numbers) which is unbounded from above; $\phi_{*}(u)=|u|^{*} \operatorname{sgn} u ; a, b \in \mathbb{T}_{1}, b>a$, $\mathbb{T}_{1}$ is another time scale; $\gamma:[a, b]_{\mathbb{T}_{1}} \rightarrow \mathbb{R}$ is a strictly increasing right-dense continuous function satisfying $0<\gamma(a)<\alpha<\gamma(b) ; p, q, e:\left[t_{0}, \infty\right)_{\mathbb{T}} \rightarrow \mathbb{R}$ are right-dense continuous with $p>0 ; r:\left[t_{0}, \infty\right)_{\mathbb{T}} \times[a, b]_{\mathbb{T}_{1}} \rightarrow \mathbb{R}$ is right-dense continuous; $\tau:\left[t_{0}, \infty\right)_{\mathbb{T}} \rightarrow\left[t_{0}, \infty\right)_{\mathbb{T}}, g:$ $\left[t_{0}, \infty\right)_{\mathbb{T}} \times[a, b]_{\mathbb{T}_{1}} \rightarrow\left[t_{0}, \infty\right)_{\mathbb{T}}$ are right-dense continuous functions satisfying $\lim _{t \rightarrow \infty} \tau(t)=$ $\lim _{t \rightarrow \infty} g(t, s)=\infty ; \xi:[a, b]_{\mathbb{T}_{1}} \rightarrow \mathbb{R}$ is strictly increasing. Here $\int_{a}^{\sigma(b)} f(s) \Delta \xi(s)$ denotes the Riemann-Stieltjes integral of the function $f$ on $[a, \sigma(b)]_{\mathbb{T}_{1}}$ with respect to $\xi, \sigma:\left[t_{0}, \infty\right)_{\mathbb{T}} \rightarrow$ $\left[t_{0}, \infty\right)_{\mathbb{T}}$ is the forward jump operator.

We restrict our attention to those solutions of (1.1) which exist on the time scale halfline $\left[T_{x}, \infty\right)_{\mathbb{T}}$, where $T_{x} \geq t_{0}$ may depend on the particular solution, a nontrivial function in any neighborhood of infinity. As usual, such a solution of (1.1) is said to be oscillatory if it is neither eventually positive nor eventually negative. Equation (1.1) is said to be oscillatory if every proper solution is oscillatory.

Recently, people have been interested in the combined effects of linear, superlinear, sublinear terms, and a forced term in oscillation. For instance, Sun and Wong [4] investigated the following forced differential equation with mixed nonlinearities

$$
\left(p(t) x^{\prime}(t)\right)^{\prime}+q(t) x(t)+\sum_{j=1}^{n} q_{j}(t) \phi_{\alpha_{j}}(x(t))=e(t)
$$

where $p, q, q_{j}, e \in C\left[t_{0}, \infty\right)$, and $0<\alpha_{1}<\cdots<\alpha_{m}<1<\alpha_{m+1}<\cdots<\alpha_{n}$. The authors obtained interval oscillation criteria for (1.2) by using an arithmetic-geometric inequality and employing arguments developed earlier in [5-9]. Sun and Meng [10] studied (1.2) again by making use of some of the arguments developed by Kong [11]. In [12], Agarwal and Zafer extended the results in [4] to dynamic equations on time scales of the form

$$
\left[p(t) \phi_{\alpha}\left(x^{\Delta}(t)\right)\right]^{\Delta}+q(t) \phi_{\alpha}(x(t))+\sum_{j=1}^{n} q_{j}(t) \phi_{\beta_{j}}(x(t))=e(t)
$$

where $t \in\left[t_{0}, \infty\right)_{\mathbb{T}}$,

$$
\beta_{1}>\beta_{2}>\cdots>\beta_{m}>\alpha>\beta_{m+1}>\cdots>\beta_{n}
$$

Very recently, Agarwal et al. [13] further to extend the results in [12] to the case of several delays of the form

$$
\left[p(t) \phi_{\alpha}\left(x^{\Delta}(t)\right)\right]^{\Delta}+q(t) \phi_{\alpha}(x(\tau(t)))+\sum_{j=1}^{n} q_{j}(t) \phi_{\beta_{j}}\left(x\left(\tau_{j}(t)\right)\right)=e(t)
$$


where $\tau(t)$ and $\tau_{j}(t)$ are right-dense continuous functions satisfying $\lim _{t \rightarrow \infty} \tau(t)=$ $\lim _{t \rightarrow \infty} \tau_{j}(t)=\infty$ for $j=1,2, \ldots, n$. Sun and Kong [14] studied the oscillation of the secondorder forced differential equation with the nonlinearity given by a Riemann-Stieltjes integral of the form

$$
\left(p(t) x^{\prime}(t)\right)^{\prime}+q(t) x(t)+\int_{0}^{b} r(t, s) \phi_{\gamma(s)}(x(\tau(s))) d \xi(s)=e(t)
$$

where $t \geq 0, \gamma \in C[0, b)$ is a strictly increasing function satisfying $0 \leq \gamma(0)<1<\gamma(b-)$. Some interval oscillation criteria of the El-Sayed type and the Kong type are established which unify many existing results in the literature.

It is obvious that (1.2), (1.3), (1.5), and (1.6) are special cases of (1.1). Some other particular cased of (1.1) can be found in [15-20]. In this paper, we will establish interval oscillation criteria for the more general (1.1). Clearly, our work is of significance because (1.1) allows an infinite number of nonlinear terms and even a continuum of nonlinearities determined by the function $\xi$. Moreover, even for the special cases of (1.2), (1.3), (1.5), and (1.6), our results generalize many existing oscillation criteria in the literature.

This paper is organized as follows. We present some lemmas in Section 2 which play a key role in the proof of the main results. The main results are given in Section 3. Two examples are given to illustrate the main results in Section 4.

\section{Preliminaries}

We here present four lemmas which play a key role in the proof of the main results in the next section. In the sequel, we denote by $L_{\xi}[a, b]_{\mathbb{T}_{1}}$ the set of Riemann-Stieltjes integrable functions on $[a, \sigma(b))_{\mathbb{T}_{1}}$ with respect to $\xi$. Assume that $\gamma, \gamma^{-1} \in L_{\xi}[a, b]_{\mathbb{T}_{1}}$. Let $h=\sup \{s \in$ $\left.(a, b)_{\mathbb{T}_{1}}: \gamma(s) \leq \alpha\right\}$.

We first present the following two Lemmas 2.1, and 2.2, which generalize Lemma 2.1 and Lemma 3.1 in [14].

Lemma 2.1. Let

$$
\begin{aligned}
& m_{1}=\alpha\left(\int_{\sigma(h)}^{\sigma(b)} \gamma^{-1}(s) \Delta \xi(s)\right)\left(\int_{\sigma(h)}^{\sigma(b)} \Delta \xi(s)\right)^{-1}, \\
& m_{2}=\alpha\left(\int_{a}^{\sigma(h)} r^{-1}(s) \Delta \xi(s)\right)\left(\int_{a}^{\sigma(h)} \Delta \xi(s)\right)^{-1} .
\end{aligned}
$$

Then for any $\delta \in\left(m_{1}, m_{2}\right)$, there exists $\eta \in L_{\xi}\left([a, b]_{\mathbb{T}_{1}}\right.$ such that $\eta(s)>0$ on $[a, b]_{\mathbb{T}_{1}}$,

$$
\begin{gathered}
\int_{a}^{\sigma(b)} \gamma(s) \eta(s) \Delta \xi(s)=\alpha, \\
\int_{a}^{\sigma(b)} \eta(s) \Delta \xi(s)=\delta .
\end{gathered}
$$


Proof. By the choice of $h$ and the definitions of $m_{1}$ and $m_{2}$, we have that $0<m_{1}<1<m_{2}$. Set

$$
\begin{aligned}
& \eta_{1}(s)= \begin{cases}\alpha \gamma^{-1}(s)\left[\int_{\sigma(h)}^{\sigma(b)} \Delta \xi(s)\right]^{-1}, & s \in[\sigma(h), b]_{\mathbb{T}}, \\
0, & s \in[a, \sigma(h))_{\mathbb{T}},\end{cases} \\
& \eta_{2}(s)= \begin{cases}0, & s \in[\sigma(h), b]_{\mathbb{T}}, \\
\alpha \gamma^{-1}(s)\left[\int_{a}^{\sigma(h)} \Delta \xi(s)\right]^{-1}, & s \in[a, \sigma(h))_{\mathbb{T}} .\end{cases}
\end{aligned}
$$

It is easy to see that $\eta_{i} \in L_{\xi}[a, b]_{\mathbb{T}_{1}}$ and

$$
\int_{a}^{\sigma(b)} \gamma(s) \eta_{i}(s) \Delta \xi(s)=\alpha, \quad i=1,2
$$

Moreover,

$$
\int_{a}^{\sigma(b)} \eta_{1}(s) \Delta \xi(s)=m_{1}, \quad \int_{a}^{\sigma(b)} \eta_{2}(s) \Delta \xi(s)=m_{2} .
$$

Let

$$
\eta(s, l)=(1-l) \eta_{1}(s)+l \eta_{2}(s), \quad s \in[a, b]_{\mathbb{T} 1}, l \in[0,1]
$$

Then we have that

$$
\begin{aligned}
& \int_{a}^{\sigma(b)} \eta(s, 0) \Delta \xi(s)=\int_{a}^{\sigma(b)} \eta_{1}(s) \Delta \xi(s)=m_{1}<1 \\
& \int_{a}^{\sigma(b)} \eta(s, 1) \Delta \xi(s)=\int_{a}^{\sigma(b)} \eta_{2}(s) \Delta \xi(s)=m_{2}>1 .
\end{aligned}
$$

By the continuous dependence of $\eta(s, l)$ on $l$, there exists $l^{*} \in(0,1)$ such that $\eta(s):=\eta\left(s, l^{*}\right)$ satisfies $\int_{a}^{\sigma(b)} \eta(s) \Delta \xi(s)=\delta \in\left(m_{1}, m_{2}\right)$. Note that $\eta(s)>0$ on $[a, b]_{\mathbb{T}_{1}}$ and $\int_{a}^{\sigma(b)} \gamma(s) \eta(s) \Delta \xi(s)=$ $\alpha$. This completes the proof of Lemma 2.1.

The next lemma is a generalized arithmetic-geometric mean inequality on time scales.

Lemma 2.2. Assume that $u:[a, b]_{\mathbb{T}_{1}} \rightarrow R$ is right-dense continuous, $\eta \in L_{\xi}[a, b]_{\mathbb{T}}, u>0, \eta>0$ on $[a, b]_{\mathbb{T}_{1}}$ and $\int_{a}^{\sigma(b)} \eta(s) \Delta \xi(s)=1$. Then

$$
\int_{a}^{\sigma(b)} \eta(s) u(s) \Delta \xi(s) \geq \exp \left(\int_{a}^{\sigma(b)} \eta(s) \ln u(s) \Delta \xi(s)\right) .
$$


Proof. Define an operator $L$ as follows:

$$
L(f)=\int_{a}^{\sigma(b)} \eta(s) f(s) \Delta \xi(s)
$$

It is obvious that $L$ is a linear operator satisfying that $L(1)=1$ and $L(u)>0$. To derive inequality (2.9) it suffices to show that

$$
L(u) \geq \exp (L(\ln u))
$$

Note that $\ln t \leq t-1$ for $t>0$. Thus, for any $s \in[a, b]_{\mathbb{T}_{1}}$ we have

$$
\ln \left(\frac{u(s)}{L(u)}\right) \leq \frac{u(s)}{L(u)}-1
$$

which follows that

$$
\ln u(s)-\ln L(u) \leq \frac{u(s)}{L(u)}-1
$$

Taking the operator $L$ on both sides of (2.13), we get

$$
\begin{aligned}
L(\ln u)-\ln L(u) & =L(\ln u-\ln L(u)) \\
& \leq L\left(\frac{u}{L(u)}\right)-L(1) \\
& =1-1=0,
\end{aligned}
$$

which implies (2.11). This completes the proof.

The following two lemmas generalize Lemma 2.4 and Lemma 6.1 in [13].

Lemma 2.3. Let $\tau:\left[t_{0}, \infty\right]_{\mathbb{T}} \rightarrow\left[t_{0}, \infty\right]_{\mathbb{T}}$ be a right-dense continuous function satisfying $0 \leq \tau(t) \leq$ $t, c, d \in\left[t_{0}, \infty\right)_{\mathbb{T}}$ with $c<d$, and $\tau_{c d}=\min \left\{\tau(t): t \in[c, d]_{\mathbb{T}}\right\}$. Assume $x:\left[\tau_{c d}, d\right]_{\mathbb{T}} \rightarrow \mathbb{R}$ is $a$ positive right-dense continuous function such that $p(t) \phi_{\alpha}\left(x^{\Delta}(t)\right)$ is nonincreasing on $\left[\tau_{c d}, d\right]_{\mathbb{T}}$. Then

$$
\frac{x(\tau(t))}{x(\sigma(t))} \geq \frac{P\left(\tau(t), \tau_{c d}\right)}{P\left(\sigma(t), \tau_{c d}\right)}, \quad t \in[c, d)
$$

where $P(t, s)=\int_{s}^{t} p^{-1 / \alpha}(s) \Delta s$. 
Proof. Set $z(t)=p^{1 / \alpha}(t) x^{\Delta}(t)$. It is not difficult to verify that $z(t)$ is nonincreasing on $\left[\tau_{c d}, d\right]_{\mathbb{T}}$ since $p(t) \phi_{\alpha}\left(x^{\Delta}(t)\right)$ is nonincreasing on $\left[\tau_{c d}, d\right]_{\mathbb{T}}$. Then we have

$$
\begin{aligned}
x(t) & =x\left(\tau_{c d}\right)+\int_{\tau_{c d}}^{t} x^{\Delta}(s) \Delta s \\
& =x\left(\tau_{c d}\right)+\int_{\tau_{c d}}^{t} p^{-1 / \alpha}(s) z(s) \Delta s \\
& \geq z(t) \int_{\tau_{c d}}^{t} p^{-1 / \alpha}(s) \Delta s \\
& =p^{1 / \alpha}(t) P\left(t, \tau_{c d}\right) x^{\Delta}(t), \quad t \in\left[\tau_{c d}, b\right]_{\mathbb{T}} .
\end{aligned}
$$

Next, for $s \in[\tau(t), \sigma(t)]_{\mathbb{T}}$, and $t \in[c, d)_{\mathbb{T}}$ we define

$$
\epsilon(s):=x(s)-p^{1 / \alpha}(s) P\left(s, \tau_{c d}\right) x^{\Delta}(s)
$$

Then (2.16) yields that $\epsilon(s) \geq 0$ for $s \in[\tau(t), \sigma(t)]_{\mathbb{T}}$ and $t \in[c, d)_{\mathbb{T}}$. Consequently, for $t \in$ $[c, d)_{\mathbb{T}}$, we have

$$
\begin{aligned}
0 \leq \int_{\tau(t)}^{\sigma(t)} \frac{p^{-1 / \alpha}(s) \epsilon(s)}{x(s) x^{\sigma}(s)} \Delta s & =\int_{\tau(t)}^{\sigma(t)}\left[\frac{P\left(s, \tau_{c d}\right)}{x(s)}\right]^{\Delta} \Delta s \\
& =\frac{P\left(\sigma(t), \tau_{c d}\right)}{x(\sigma(t))}-\frac{P\left(\tau(t), \tau_{c d}\right)}{x(\tau(t))}
\end{aligned}
$$

This implies (2.15). The proof of Lemma 2.3 is complete.

Similar to the proof of Lemma 2.3, we can get the following result.

Lemma 2.4. Let $\tau:\left[t_{0}, \infty\right]_{\mathbb{T}} \rightarrow\left[t_{0}, \infty\right]_{\mathbb{T}}$ be a right-dense continuous function satisfying $\tau(t)>t$, $c, d \in\left[t_{0}, \infty\right)_{\mathbb{T}}$ with $c<d$, and $\tau^{c d}=\max \left\{\tau(t): t \in[c, d]_{\mathbb{T}}\right\}$. If $x:\left[c, \tau^{c d}\right]_{\mathbb{T}} \rightarrow \mathbb{R}$ is a positive right-dense continuous function for which $p(t) \phi_{\alpha}\left(x^{\Delta}(t)\right)$ is nonincreasing on $\left[c, \tau^{c d}\right]_{\mathbb{T}}$, then

$$
\frac{x(\tau(t))}{x(\sigma(t))} \geq \frac{P\left(\tau^{c d}, \tau(t)\right)}{P\left(\tau^{c d}, \sigma(t)\right)}, \quad t \in[c, d)_{\mathbb{T}}
$$

where $P(t, s)$ is defined as in Lemma 2.3.

\section{Main Results}

We note from the definition of $m_{1}$ and $m_{2}$ that $0<m_{1}<1<m_{2}$. In the following we will use the values of $\delta$ in the interval $\left(m_{1}, 1\right]$ to establish interval criteria for oscillation of (1.1). For $c, d \in\left[t_{0}, \infty\right)_{\mathbb{T}}$ with $c<d$, we define the function class $\mathcal{u}(c, d)=\left\{u \in C_{\mathrm{rd}}^{1}[c, d]_{\mathbb{T}}: u(c)=0=\right.$ $u(d), u \neq 0\}$, where $C_{\mathrm{rd}}^{1}[c, d]_{\mathbb{T}}$ denotes the set of right-dense continuously $\Delta$-differentiable 
functions on $[c, d]_{\mathbb{T}}$. In the following, let $\tau_{c d}$ and $\tau^{c d}$ be defined as in Lemmas 2.3 and 2.4. Set

$$
g_{c d}=\min _{(t, s) \in[c, d]_{\mathbb{T}} \times[a, b]_{\mathbb{T}_{1}}} g(t, s), \quad g^{c d}=\max _{(t, s) \in[c, d]_{\mathbb{T}} \times[a, b]_{\mathbb{T}}} g(t, s) .
$$

Theorem 3.1. Assume that $\tau(t), g(t, s) \leq t$ for $t \in\left[t_{0}, \infty\right)_{\mathbb{T}}$ and $s \in[a, b]_{\mathbb{T}_{1}}$. Suppose also that for any $T \geq t_{0}$, there exist subintervals $\left[c_{i}, d_{i}\right]_{\mathbb{T}}$ of $[T, \infty), i=1,2$, such that $c_{i}, d_{i} \in \mathbb{T}, d_{i}>c_{i}$, and

$$
\begin{gathered}
r(t, s) \geq 0, \quad(t, s) \in\left[h_{i}, d_{i}\right)_{\mathbb{T}} \times[a, b]_{\mathbb{T} 1}, \\
(-1)^{i} e(t) \geq 0, \quad t \in\left[h_{i}, d_{i}\right)_{\mathbb{T}}
\end{gathered}
$$

where $h_{i}=\min \left\{\tau_{c_{i}} d_{i}, g_{c_{i}} d_{i}\right\}$. For each $\delta \in\left(m_{1}, 1\right]$, let $\eta \in L_{\xi}[a, b]_{\mathbb{T}_{1}}$ be defined as in Lemma 2.1. If there exists $u_{i} \in \mathfrak{U}\left(c_{i}, d_{i}\right)$ for $i=1,2$ such that

$$
\sup _{\delta \in\left(m_{1}, 1\right]} \int_{c_{i}}^{d_{i}}\left[Q_{i}(t)\left|u_{i}(\sigma(t))\right|^{\alpha+1}-p(t)\left|u_{i}^{\Delta}(t)\right|^{\alpha+1}\right] \Delta t \geq 0
$$

where

$$
\begin{aligned}
Q_{i}(t)= & q(t)\left[\frac{P\left(\tau(t), \tau_{c_{i} d_{i}}\right)}{P\left(\sigma(t), \tau_{c_{i} d_{i}}\right)}\right]^{\alpha}+\left[\frac{|e(t)|}{1-\delta}\right]^{1-\delta} \\
& \times \exp \left(\int_{a}^{\sigma(b)} \eta(s) \ln \left(\frac{r(t, s)}{\eta(s)}\left[\frac{P\left(g(t, s), g_{c_{i} d_{i}}\right)}{P\left(\sigma(t), g_{c_{i} d_{i}}\right)}\right]^{\gamma(s)}\right) \Delta \xi(s)\right) .
\end{aligned}
$$

Here we use the convention that $\ln 0=-\infty, e^{-\infty}=0$, and $0^{1-\delta}=0$ and $(1-\delta)^{1-\delta}=1$ for $\delta=1$ due to the fact that $\lim _{t \rightarrow 0} t^{t}=1$. Then (1.1) is oscillatory.

Proof. We prove this result by the contradiction method. Assume the contrary. Then (1.1) has an extendible solution $x(t)$ which is eventually positive or negative. Without loss of generality, we may assume that $x(t)>0$ for all $t \in\left[t_{0}, \infty\right)_{\mathbb{T}}$. When $x(t)$ is eventually negative, the proof is in the same way except that the interval $\left[c_{2}, d_{2}\right]_{\mathbb{T}}$, instead of $\left[c_{1}, d_{1}\right]_{\mathbb{T}}$, is used. Define

$$
w(t)=-\frac{p(t) \phi_{\alpha}\left(x^{\Delta}(t)\right)}{\phi_{\alpha}(x(t))}, \quad t \in\left[c_{1}, d_{1}\right]_{\mathbb{T}} .
$$

It follows that

$$
\begin{aligned}
w^{\Delta}(t)= & q(t) \frac{\phi_{\alpha}(x(\tau(t)))}{\phi_{\alpha}(x(\sigma(t)))}+\int_{a}^{\sigma(b)} r(t, s) \frac{\phi_{r(s)}(x(g(t, s)))}{\phi_{\alpha}(x(\sigma(t)))} \Delta \xi(s) \\
& -\frac{e(t)}{\phi_{\alpha}(x(\sigma(t)))}+p(t) \frac{\phi_{\alpha}\left(x^{\Delta}(t)\right)\left[\phi_{\alpha}(x(t))\right]^{\Delta}}{\phi_{\alpha}(x(t)) \phi_{\alpha}(x(\sigma(t)))} .
\end{aligned}
$$


It is obvious that the conditions in Lemma 2.3 are satisfied with $\tau$ replaced by $g(t, s)$. By (2.15) we have for $t \in\left[c_{1}, d_{1}\right)_{\mathbb{T}}$

$$
\frac{x(\tau(t))}{x(\sigma(t))} \geq \frac{P\left(\tau(t), \tau_{c_{1} d_{1}}\right)}{P\left(\sigma(t), \tau_{c_{1} d_{1}}\right)}, \quad \frac{x(g(t, s))}{x(\sigma(t))} \geq \frac{P\left(g(t, s), g_{c_{1} d_{1}}\right)}{P\left(\sigma(t), g_{c_{1} d_{1}}\right)}
$$

By (3.2), (3.6), and (3.7), and the fact that $\phi_{*}$ is increasing, we get

$$
\begin{aligned}
w^{\Delta}(t) \geq & q(t)\left[\frac{P\left(\tau(t), \tau_{c_{1} d_{1}}\right)}{P\left(\sigma(t), \tau_{c_{1} d_{1}}\right)}\right]^{\alpha}-\frac{e(t)}{\phi_{\alpha}(x(\sigma(t)))} \\
& +\int_{a}^{\sigma(b)} r(t, s)\left[\frac{P\left(g(t, s), g_{c_{1} d_{1}}\right)}{P\left(\sigma(t), g_{c_{1} d_{1}}\right)}\right]^{\gamma(s)}[x(\sigma(t))]^{\gamma(s)-\alpha} \Delta \xi(s) \\
& +p(t) \frac{\phi_{\alpha}\left(x^{\Delta}(t)\right)\left[\phi_{\alpha}(x(t))\right]^{\Delta}}{\phi_{\alpha}(x(t)) \phi_{\alpha}(x(\sigma(t)))}
\end{aligned}
$$

(I) We first consider the case where the supremum in (3.3) is assumed at $\delta=1$. From (3.2) and (3.8) we have that for $t \in\left[c_{1}, d_{1}\right)_{\mathbb{T}}$

$$
\begin{aligned}
w^{\Delta}(t) \geq & q(t)\left[\frac{P\left(\tau(t), \tau_{c_{1} d_{1}}\right)}{P\left(\sigma(t), \tau_{c_{1} d_{1}}\right)}\right]^{\alpha}+p(t) \frac{\phi_{\alpha}\left(x^{\Delta}(t)\right)\left[\phi_{\alpha}(x(t))\right]^{\Delta}}{\phi_{\alpha}(x(t)) \phi_{\alpha}(x(\sigma(t)))} \\
& +\int_{a}^{\sigma(b)} r(t, s)\left[\frac{P\left(g(t, s), g_{c_{1} d_{1}}\right)}{P\left(\sigma(t), g_{c_{1} d_{1}}\right)}\right]^{\gamma(s)}[x(\sigma(t))]^{\gamma(s)-\alpha} \Delta \xi(s) .
\end{aligned}
$$

Let $\eta \in L_{\xi}[a, b]_{\mathbb{T}_{1}}$ be defined as in Lemma 2.1 with $\delta=1$. Then $\eta$ satisfies (2.2) and (2.3) with $\delta=1$. This follows that

$$
\int_{a}^{\sigma(b)} \eta(s)[\gamma(s)-\alpha] \Delta \xi(s)=0
$$

Therefore, by Lemma 2.2 we have that for $t \in\left[c_{1}, d_{1}\right]_{\mathbb{T}}$

$$
\begin{aligned}
& \int_{a}^{\sigma(b)} r(t, s)\left[\frac{P\left(g(t, s), g_{c_{1} d_{1}}\right)}{P\left(\sigma(t), g_{c_{1} d_{1}}\right)}\right]^{\gamma(s)}[x(\sigma(t))]^{\gamma(s)-\alpha} \Delta \xi(s) \\
& \quad \int_{a}^{\sigma(b)} \eta(s) \eta^{-1}(s) r(t, s)\left[\frac{P\left(g(t, s), g_{c_{1} d_{1}}\right)}{P\left(\sigma(t), g_{c_{1} d_{1}}\right)}\right]^{\gamma(s)}[x(\sigma(t))]^{\gamma(s)-\alpha} \Delta \xi(s) \\
& \quad \geq \exp \left(\int_{a}^{\sigma(b)} \eta(s) \ln \left(\frac{r(t, s)}{\eta(s)}\left[\frac{P\left(g(t, s), g_{c_{1} d_{1}}\right)}{P\left(\sigma(t), g_{c_{1} d_{1}}\right)}\right]^{\gamma(s)}[x(\sigma(t))]^{\gamma(s)-\alpha}\right) \Delta \xi(s)\right)
\end{aligned}
$$




$$
\begin{aligned}
= & \exp \left(\int_{a}^{\sigma(b)} \eta(s) \ln \left(\frac{r(t, s)}{\eta(s)}\left[\frac{P\left(g(t, s), g_{c_{1} d_{1}}\right)}{P\left(\sigma(t), g_{c_{1} d_{1}}\right)}\right]^{\gamma(s)}\right) \Delta \xi(s)\right) \\
& \times \exp \left(\ln x(\sigma(t)) \int_{a}^{\sigma(b)} \eta(s)[\gamma(s)-\alpha] \Delta \xi(s)\right) \\
= & \exp \left(\int_{a}^{\sigma(b)} \eta(s) \ln \left(\frac{r(t, s)}{\eta(s)}\left[\frac{P\left(g(t, s), g_{c_{1} d_{1}}\right)}{P\left(\sigma(t), g_{c_{1} d_{1}}\right)}\right]^{\gamma(s)}\right) \Delta \xi(s)\right) .
\end{aligned}
$$

Substituting (3.11) into (3.9) we obtain

$$
w^{\Delta}(t) \geq Q_{1}(t)+p(t) \frac{\phi_{\alpha}\left(x^{\Delta}(t)\right)\left[\phi_{\alpha}(x(t))\right]^{\Delta}}{\phi_{\alpha}(x(t)) \phi_{\alpha}(x(\sigma(t)))}, \quad t \in\left[c_{1}, d_{1}\right]_{\mathbb{T}}
$$

where $Q_{1}(t)$ is defined by (3.4) with $i=1$ and $\delta=1$. Multiplying both sides of the above inequality by $\left|u_{1}(\sigma(t))\right|^{\alpha+1}$ and proceeding as in the proof of Theorem 3.1 in [13], we can get a contradiction with (3.3).

(II) Now we consider the case where the supremum in (3.3) is assumed at $\delta \in\left(m_{1}, 1\right)$. Let $\tilde{\eta}(s)=\delta^{-1} \eta(s)$. Then from (2.2) and (2.3), we get

$$
\int_{a}^{\sigma(b)} \tilde{\eta}(s) \Delta \xi(s)=1, \quad \int_{a}^{\sigma(b)} \tilde{\eta}(s)[\delta \gamma(s)-\alpha] \Delta \xi(s)=0 .
$$

Hence for $t \in\left[c_{1}, d_{1}\right]_{\mathbb{T}}$

$$
-\frac{e(t)}{\phi_{\alpha}(x(\sigma(t)))}+\int_{a}^{\sigma(b)} r(t, s)\left[\frac{P\left(g(t, s), g_{c_{1} d_{1}}\right)}{P\left(\sigma(t), g_{c_{1} d_{1}}\right)}\right]^{\gamma(s)}[x(\sigma(t))]^{\gamma(s)-\alpha} \Delta \xi(s)=\int_{a}^{\sigma(b)} \tilde{\eta}(s) \Omega(t, s) \Delta \xi(s),
$$

where

$$
\Omega(t, s)=\delta \frac{r(t, s)}{\eta(s)}\left[\frac{P\left(g(t, s), g_{c_{1} d_{1}}\right)}{P\left(\sigma(t), g_{c_{1} d_{1}}\right)}\right]^{\gamma(s)}[x(\sigma(t))]^{\gamma(s)-\alpha}+\frac{|e(t)|}{x^{\alpha}(\sigma(t))} .
$$

On the other hand, by the basic arithmetic-geometric mean inequality, we have that

$$
\Omega(t, s) \geq\left[\frac{r(t, s)}{\eta(s)}\right]^{\delta}\left[\frac{P\left(g(t, s), g_{c_{1} d_{1}}\right)}{P\left(\sigma(t), g_{c_{1} d_{1}}\right)}\right]^{\delta \gamma(s)}\left[\frac{|e(t)|}{1-\delta}\right]^{1-\delta}[x(\sigma(t))]^{\delta \gamma(s)-\alpha} .
$$


Substituting (3.16) into (3.14), using Lemma 2.2 and similar to the computation in (I), for $t \in\left[c_{1}, d_{1}\right]_{\mathbb{T}}$ we can get

$$
\begin{gathered}
-\frac{e(t)}{\phi \alpha(x(\sigma(t)))}+\int_{a}^{\sigma(b)} r(t, s)\left[\frac{P\left(g(t, s), g_{c_{1} d_{1}}\right)}{P\left(\sigma(t) g_{c_{1} d_{1}}\right)}\right]^{\gamma(s)} x^{\gamma(s)-\alpha}(\sigma(t)) \Delta \xi(s) \\
\geq \int_{a}^{\sigma(b)} \tilde{\eta}(s)\left[\frac{r(t, s)}{\eta(s)}\right]^{\delta}\left[\frac{P\left(g(t, s), g_{c_{1} d_{1}}\right)}{P\left(\sigma(t), g_{c_{1} d_{1}}\right)}\right]^{\delta \gamma(s)}\left[\frac{|e(t)|}{1-\delta}\right]^{1-\delta}[x(\sigma(t))]^{\delta \gamma(s)-\alpha} \Delta \xi(s) \\
\geq\left[\frac{|e(t)|}{1-\delta}\right]^{1-\delta} \exp \left(\int_{a}^{\sigma(b)} \eta(s) \ln \left(\frac{r(t, s)}{\eta(s)}\left[\frac{P\left(g(t, s), g_{c_{1} d_{1}}\right)}{P\left(\sigma(t), g_{c_{1} d_{1}}\right)}\right]^{\gamma(s)}\right) \Delta \xi(s)\right)
\end{gathered}
$$

which also implies (3.12) for $t \in\left[c_{1}, d_{1}\right]_{\mathbb{T}}$. The rest of the proof is similar to Part (I) and hence is omitted. This completes the proof of Theorem 3.1.

For the case when $\tau(t), g(t, s)>t$ for $t \in\left[t_{0}, \infty\right)_{\mathbb{T}}$ and $s \in[a, b]_{\mathbb{T}_{1}}$, using Lemma 2.4 and following the proof of Theorem 3.1, we have the following oscillation result for (1.1) immediately.

Theorem 3.2. Assume that $\tau(t), g(t, s)>t$ for $t \in\left[t_{0}, \infty\right)_{\mathbb{T}}$ and $s \in[a, b]_{\mathbb{T}_{1}}$. Suppose also that for any $T \geq t_{0}$, there exist subintervals $\left[c_{i}, d_{i}\right]_{\mathbb{T}}$ of $[T, \infty), i=1,2$, such that $c_{i}, d_{i} \in \mathbb{T}, d_{i}>c_{i}$, and (3.2) holds for $t \in\left[c_{i}, \bar{h}_{i}\right)_{\mathbb{T}}$ and $s \in[a, b]_{\mathbb{T}}$, where $\bar{h}_{i}=\max \left\{\tau^{c_{i} d_{i}}, g^{c_{i} d_{i}}\right\}$. For each $\delta \in\left(m_{1}, 1\right]$, let $\eta \in L_{\xi}[a, b]_{\mathbb{T}_{1}}$ be defined as in Lemma 2.1. If there exists $u_{i} \in \mathcal{U}\left(c_{i}, d_{i}\right)$ for $i=1,2$ such that (3.4) holds, where

$$
\begin{aligned}
Q_{i}(t)= & q(t)\left[\frac{P\left(\tau^{c_{1} d_{1}}, \tau(t)\right)}{P\left(\tau^{c_{1} d_{1}}, \sigma(t)\right)}\right]^{\alpha}+\left[\frac{|e(t)|}{1-\delta}\right]^{1-\delta} \\
& \times \exp \left(\int_{a}^{\sigma(b)} \eta(s) \ln \left(\frac{r(t, s)}{\eta(s)}\left[\frac{P\left(g^{c_{1} d_{1}}, g(t, s)\right)}{P\left(g^{c_{1} d_{1}}, \sigma(t)\right)}\right]^{\gamma(s)}\right) \Delta \xi(s)\right) .
\end{aligned}
$$

Then (1.1) is oscillatory.

Remark 3.3. We see from the proof of Lemma 2.1 in Section 2 that for each $\delta \in\left(m_{1}, 1\right]$, the function $\eta$ can be constructed explicitly for any nondecreasing function $\xi$, and hence the functions $Q_{i}$ in Theorems 3.1 and 3.2 are explicitly given.

Remark 3.4. We observe that in Theorems 3.1 and 3.2, if the supremum in (3.3) is assumed at $\delta=1$, the effect of $e(t)$ is neglected in some extent. This implies that the magnitude of $e(t)$ in $\left[c_{i}, d_{i}\right]_{\mathbb{T}}$ cannot be large. For otherwise, the supremum would have been taken at some $\delta \in\left(m_{1}, 1\right)$. 
Now, we interpret the results for (1.1) to the special case of (1.5). Set $\mathbb{T}_{1}=\mathbb{N}, a=1$, $b=n+1$ for $n \in \mathbb{N}$, and

$$
\begin{gathered}
\xi(s)=s, \quad s=1,2, \ldots, n+1, \\
r(s)=\beta_{s} \text { satisfying }(1.4), \quad s=1,2, \ldots, n, \\
r(t, s)=q_{s}(t), \quad s=1,2, \ldots, n, \\
g(t, s)=\tau_{s}(t), \quad s=1,2, \ldots, n .
\end{gathered}
$$

Then (1.1) reduces to (1.5). By a straightforward computation, we have that

$$
m_{1}=\frac{\alpha}{n-m} \sum_{j=m+1}^{n} \beta_{j}^{-1}, \quad m_{2}=\frac{\alpha}{m} \sum_{j=1}^{m} \beta_{j}^{-1}
$$

Then Lemma 2.1 can be restated as the following: for any $\delta \in\left(m_{1}, m_{2}\right)$, there exists a positive $n$-tuple $\left(\eta_{1}, \ldots, \eta_{n}\right)$ satisfying

$$
\sum_{j=1}^{n} \alpha_{j} \eta_{j}=\alpha, \quad \sum_{j=1}^{n} \eta_{j}=\delta
$$

Therefore, by Theorems 3.1 and 3.2, we obtain the following oscillation results for (1.5) which generalize the results in [13].

Corollary 3.5. Assume that $\tau(t), \tau_{j}(t) \leq t$ for $t \in\left[t_{0}, \infty\right)_{\mathbb{T}}$ and $j=1, \ldots, n$. Suppose also that for any $T \geq t_{0}$, there exist subintervals $\left[c_{i}, d_{i}\right]_{\mathbb{T}}$ of $[T, \infty), i=1,2$, such that $c_{i}, d_{i} \in \mathbb{T}, d_{i}>c_{i}$, and

$$
q_{j}(t) \geq 0, \quad(-1)^{i} e(t) \geq 0, \quad t \in\left[\theta_{i}, d_{i}\right)_{\mathbb{T}},
$$

where $\theta_{i}=\min \left\{\omega_{i}, \omega_{i j}: j=1,2, \ldots, n\right\}, \omega_{i}=\min \left\{\tau(t): t \in\left[c_{i}, d_{i}\right]_{\mathbb{T}}\right\}$ and $\omega_{i j}=\min \left\{\tau_{j}(t): t \in\right.$ $\left.\left[c_{i}, d_{i}\right]_{\mathbb{T}}\right\}$. For each $\delta \in\left(m_{1}, 1\right]$, let $\left(\eta_{1}, \ldots, \eta_{n}\right)$ be defined by (3.21). We further assume that there exists a function $u_{i} \in \mathfrak{U}\left(a_{i}, b_{i}\right)$ such that (3.4) holds, where

$$
Q_{i}(t)=q(t)\left[\frac{P\left(\tau(t), \omega_{i}\right)}{P\left(\sigma(t), \omega_{i}\right)}\right]^{\alpha}+\left[\frac{|e(t)|}{1-\delta}\right]^{1-\delta} \prod_{j=1}^{n}\left[\frac{q_{j}(t)}{\eta_{j}}\right]^{\eta_{j}}\left[\frac{P\left(\tau_{j}(t), \omega_{i j}\right)}{P\left(\sigma(t), \omega_{i j}\right)}\right]^{\beta_{j} \eta_{j}}
$$

Then (1.5) is oscillatory.

Corollary 3.6. Assume that $\tau(t), \tau_{j}(t)>t$ for $t \in\left[t_{0}, \infty\right)_{\mathbb{T}}$ and $j=1, \ldots, n$. Suppose also that for any $T \geq t_{0}$, there exist subintervals $\left[c_{i}, d_{i}\right]_{\mathbb{T}}$ of $[T, \infty), i=1,2$, such that $c_{i}, d_{i} \in \mathbb{T}, d_{i}>c_{i}$, and

$$
q_{j}(t) \geq 0, \quad(-1)^{i} e(t) \geq 0, \quad t \in\left[c_{i}, \bar{\theta}_{i}\right)_{\mathbb{T}^{\prime}}
$$


where $\bar{\theta}_{i}=\max \left\{\bar{\omega}_{i}, \bar{\omega}_{i j}: j=1,2, \ldots, n\right\}, \bar{\omega}_{i}=\max \left\{\tau(t): t \in\left[c_{i}, d_{i}\right]_{\mathbb{T}}\right\}$ and $\bar{\omega}_{i j}=\max \left\{\tau_{j}(t): t \in\right.$ $\left.\left[c_{i}, d_{i}\right]_{\mathbb{T}}\right\}$. For each $\delta \in\left(m_{1}, 1\right]$, let $\left(\eta_{1}, \ldots, \eta_{n}\right)$ be defined by (3.21). We further assume there exists a function $u_{i} \in \mathfrak{U}\left(a_{i}, b_{i}\right)$ such that (3.4) holds, where

$$
Q_{i}(t)=q(t)\left[\frac{P\left(\bar{\omega}_{i}, \tau(t)\right)}{P\left(\bar{\omega}_{i}, \sigma(t)\right)}\right]^{\alpha}+\left[\frac{|e(t)|}{1-\delta}\right]^{1-\delta} \prod_{j=1}^{n}\left[\frac{q_{j}(t)}{\eta_{j}}\right]^{\eta_{j}}\left[\frac{P\left(\bar{\omega}_{i j}, \tau_{j}(t)\right)}{P\left(\bar{\omega}_{i j}, \sigma(t)\right)}\right]^{\beta_{j} \eta_{j}}
$$

Then (1.5) is oscillatory.

Remark 3.7. Corollaries 3.5 and 3.6 generalize those results in [13] since the sufficient condition for oscillation of (1.5) is given here in the form of $\sup _{\delta \in\left(m_{1}, 1\right]} \int_{c_{i}}^{d_{i}}[\cdot] \Delta t \geq 0$.

\section{Examples}

We will give two examples to illustrate Theorems 3.1 and 3.2 in the case when $\xi(s)=s, \mathbb{T}_{1}=\mathbb{R}$, and $\mathbb{T}=\mathbb{R}(\mathbb{N})$.

Example 4.1. Consider on $\mathbb{T}=\mathbb{R}$ the following differential equation

$$
\left(\phi_{3 / 2}\left(x^{\prime}(t)\right)\right)^{\prime}+k_{1} \sin t \phi_{3 / 2} x\left(t-\frac{\pi}{4}\right)+k_{2} \sin t \int_{1}^{2} \phi_{s}(x(t)) d s=-f(t) \cos t
$$

where $t \geq 0, k_{1}, k_{2}>0$ are constants, $f \in C[0, \infty)$ is an arbitrary nonnegative function. Here we have $p(t)=1, q(t)=k_{1} \sin t, r(t, s)=k_{2} \sin t, e(t)=-f(t) \cos t, a=1, b=2, \gamma(s)=s$, $\tau(t)=t-2 \pi$, and $g(t, s)=t$. For any $T \geq 0$, we choose $k$ large enough so that $2 k \pi \geq T$ and let $c_{1}=2 k \pi+\pi / 4, d_{1}=2 k \tau+\pi / 2, c_{2}=2 k \pi+3 \pi / 4$ and $d_{2}=2 k \pi+\pi$. Then it is easy to verify that (3.2) holds, and

$$
Q_{i}(t)=k_{1} \sin t\left(\frac{t-c_{i}}{t-c_{i}+(\pi / 4)}\right)^{3 / 2}+k_{2} \sin t, \quad i=1,2
$$

A straightforward computation yields that $m_{1}=3 \ln (4 / 3)=0.863$ and $m_{2}=3 \ln (3 / 2)=$ 1.2164. By Lemma 2.1, for any $\delta \in\left(m_{1}, m_{2}\right)$, there exists a positive Riemann integrable function on [1,2] such that (2.2) and (2.3) hold. Particularly, we can choose $\delta=1$ and hence $\eta(s)=1$. If we choose $u_{i}(t)=\left(t-c_{i}\right)\left(d_{i}-t\right)$ for $i=1,2$, then we have

$$
\begin{aligned}
& \int_{c_{i}}^{d_{i}} Q_{i}(t)\left|u_{i}(t)\right|^{5 / 2} d t \\
& \quad=\int_{\pi / 4}^{\pi / 2} \sin t\left[k_{1} \frac{(t-(\pi / 4))^{4}((\pi / 2)-t)^{5 / 2}}{t^{3 / 2}}+k_{2}\left(\left(t-\frac{\pi}{4}\right)\left(\frac{\pi}{2}-t\right)\right)^{5 / 2}\right] d t \\
& \int_{c_{i}}^{d_{i}}\left|u_{i}^{\prime}(t)\right|^{5 / 2} d t=\int_{\pi / 4}^{\pi / 2}\left|\frac{3 \pi}{4}-2 t\right|^{5 / 2} d t=\frac{2}{7}\left(\frac{\pi}{4}\right)^{7 / 2} .
\end{aligned}
$$


If there exist positive constants $k_{1}$ and $k_{2}$ such that

$$
\int_{c_{i}}^{d_{i}} Q_{i}(t)\left|u_{i}(t)\right|^{5 / 2} d t \geq \frac{2}{7}\left(\frac{\pi}{4}\right)^{7 / 2}
$$

then condition (3.3) holds. By Theorem 3.1, we have that (4.1) is oscillatory.

Example 4.2. Consider on $\mathbb{T}=\mathbb{N}$ the following difference equation

$$
\left(\phi_{3 / 2}\left(x^{\Delta}(t)\right)\right)^{\Delta}+q(t) \phi_{3 / 2}(x(t+1))+r(t) \int_{1}^{2} \phi_{s}(x(t+1)) d s=e(t),
$$

where $t \in \mathbb{N}$,

$$
\begin{gathered}
q(t)= \begin{cases}k_{1}, & t=8 j, 8 j+1,8 j+2,8 j+3,8 j+4,8 j+5, \\
f_{1}(t), & t=8 j+6,8 j+7,\end{cases} \\
r(t)= \begin{cases}k_{2}, & t=8 j, 8 j+1,8 j+2,8 j+3,8 j+4,8 j+5, \\
f_{2}(t), & t=8 j+6,8 j+7,\end{cases} \\
e(t)= \begin{cases}f_{3}(t), & t=8 j, 8 j+1,8 j+2, \\
f_{4}(t), & t=8 j+3,8 j+4,8 j+5, \\
f_{5}(t), & t=8 j+6,8 j+7,\end{cases}
\end{gathered}
$$

for $j \in \mathbb{N}, k_{1}$ and $k_{2}$ are positive constants, $f_{i}(t)(i=1,2,3,4,5)$ are arbitrary real-valued functions with $f_{3}(t) \leq 0$ and $f_{4}(t) \geq 0$. For any $N \in \mathbb{N}$, we can choose $j$ large enough so that $8 j \geq N$. Let $c_{1}=8 j+1, d_{1}=8 j+3, c_{2}=8 j+4$, and $d_{2}=8 j+6$. Then (3.2) is valid. Choose $\eta(s)=1$ and $u_{i}(t)=\left(t-c_{i}\right)\left(d_{i}-t\right)$ for $i=1,2$. Then (2.2) and (2.3) hold with $\delta=1$. By the straightforward computation, we get

$$
\int_{c_{i}}^{d_{i}}\left[Q_{i}(t)\left|u_{i}(\sigma(t))\right|^{5 / 2}-\left|u_{i}^{\Delta}(t)\right|^{5 / 2}\right] \Delta t=k_{1}+k_{2}-1
$$

By Theorem 3.2, (4.5) is oscillatory if $k_{1}+k_{2} \geq 1$.

\section{Acknowledgments}

The author thanks the reviewer for his/her valuable comments on this paper. This paper was supported by the national natural science foundations of China $(60704039,61174217)$ and the natural science foundations of Shandong Province (ZR2010AL002, JQ201119). 


\section{References}

[1] S. Hilger, "Analysis on measure chains-A unified approach to continuous and discrete calculus," Results in Mathematics, vol. 18, no. 1-2, pp. 18-56, 1990.

[2] M. Bohner and A. Peterson, Dynamic Equations on Time Scales, Birkhäuser, Boston, Mass, USA, 2001.

[3] M. Bohner and A. Peterson, Eds., Advances in Dynamic Equations on Time Scales, Birkhäuser, Boston, Mass, USA, 2003.

[4] Y. G. Sun and J. S. W. Wong, "Oscillation criteria for second order forced ordinary differential equations with mixed nonlinearities," Journal of Mathematical Analysis and Applications, vol. 334, no. 1, pp. 549-560, 2007.

[5] M. A. El-Sayed, "An oscillation criterion for a forced second order linear differential equation," Proceedings of the American Mathematical Society, vol. 118, no. 3, pp. 813-817, 1993.

[6] Y. G. Sun and J. S. W. Wong, "Note on forced oscillation of $n$ th-order sublinear differential equations," Journal of Mathematical Analysis and Applications, vol. 298, no. 1, pp. 114-119, 2004.

[7] A. H. Nasr, "Sufficient conditions for the oscillation of forced super-linear second order differential equations with oscillatory potential," Proceedings of the American Mathematical Society, vol. 126, no. 1, pp. 123-125, 1998.

[8] Ch. G. Philos, "Oscillation theorems for linear differential equations of second order," Archiv der Mathematik, vol. 53, no. 5, pp. 482-492, 1989.

[9] Y. G. Sun, "A note on Nasr's and Wong's papers," Journal of Mathematical Analysis and Applications, vol. 286, no. 1, pp. 363-367, 2003.

[10] Y. G. Sun and F. W. Meng, "Interval criteria for oscillation of second-order differential equations with mixed nonlinearities," Applied Mathematics and Computation, vol. 198, no. 1, pp. 375-381, 2008.

[11] Q. Kong, "Interval criteria for oscillation of second-order linear ordinary differential equations," Journal of Mathematical Analysis and Applications, vol. 229, no. 1, pp. 258-270, 1999.

[12] R. P. Agarwal and A. Zafer, "Oscillation criteria for second-order forced dynamic equations with mixed nonlinearities," Advances in Difference Equations, vol. 2009, Article ID 938706, 20 pages, 2009.

[13] R. P. Agarwal, D. R. Anderson, and A. Zafer, "Interval oscillation criteria for second-order forced delay dynamic equations with mixed nonlinearities," Computers $\mathcal{E}$ Mathematics with Applications, vol. 59, no. 2, pp. 977-993, 2010.

[14] Y. Sun and Q. Kong, "Interval criteria for forced oscillation with nonlinearities given by RiemannStieltjes integrals," Computers and Mathematics with Applications, vol. 62, no. 1, pp. 243-252, 2011.

[15] Y. G. Sun and F. W. Meng, “Oscillation of second-order delay differential equations with mixed nonlinearities," Applied Mathematics and Computation, vol. 207, no. 1, pp. 135-139, 2009.

[16] D. R. Anderson, "Oscillation of second-order forced functional dynamic equations with oscillatory potentials," Journal of Difference Equations and Applications, vol. 13, no. 5, pp. 407-421, 2007.

[17] A. Zafer, "Interval oscillation criteria for second order super-half linear functional differential equations with delay and advanced arguments," Mathematische Nachrichten, vol. 282, no. 9, pp. 1334-1341, 2009.

[18] D. R. Anderson and A. Zafer, "Interval criteria for second-order super-half-linear functional dynamic equations with delay and advance arguments," Journal of Difference Equations and Applications, vol. 16, no. 8, pp. 917-930, 2010.

[19] S. Sun, T. Li, Z. Han, and Y. Sun, "Oscillation of second-order neutral functional differential equations with mixed nonlinearities," Abstract and Applied Analysis, vol. 2011, Article ID 927690, 15 pages, 2011.

[20] Y. Bai and L. Liu, "New oscillation criteria for second-order delay differential equations with mixed nonlinearities," Discrete Dynamics in Nature and Society, vol. 2010, Article ID 796256, 2010 pages, 2010. 


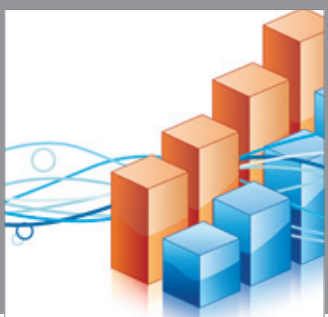

Advances in

Operations Research

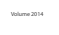

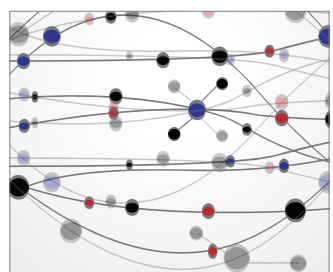

\section{The Scientific} World Journal
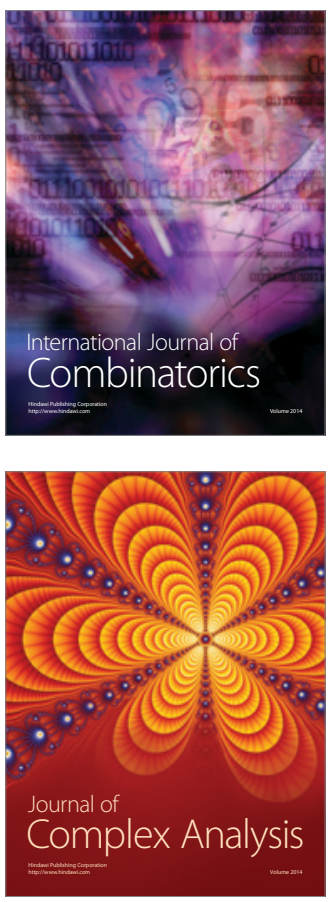

International Journal of

Mathematics and

Mathematical

Sciences
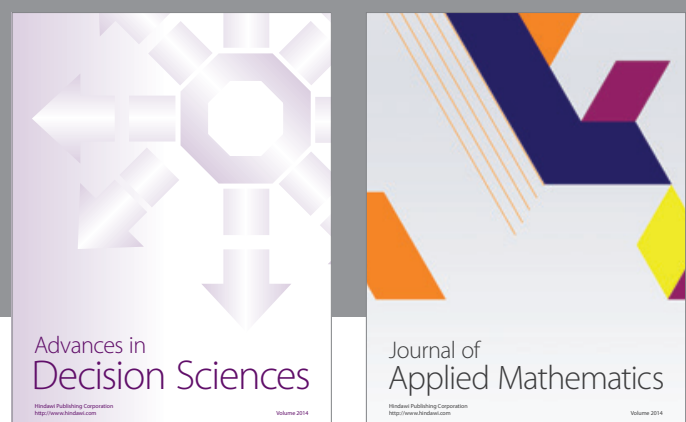

Journal of

Applied Mathematics
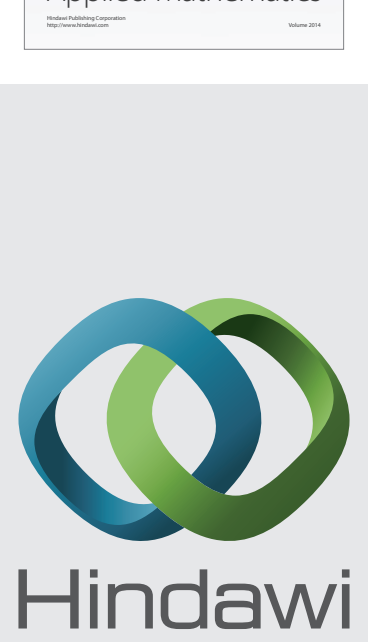

Submit your manuscripts at http://www.hindawi.com
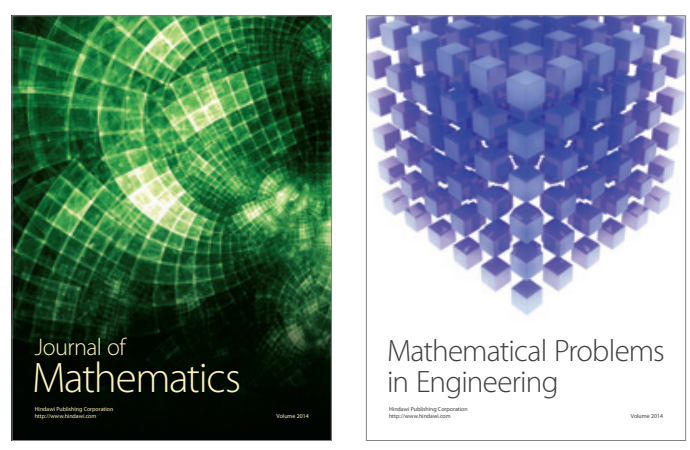

Mathematical Problems in Engineering
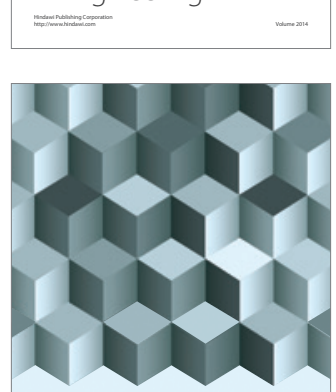

Journal of

Function Spaces
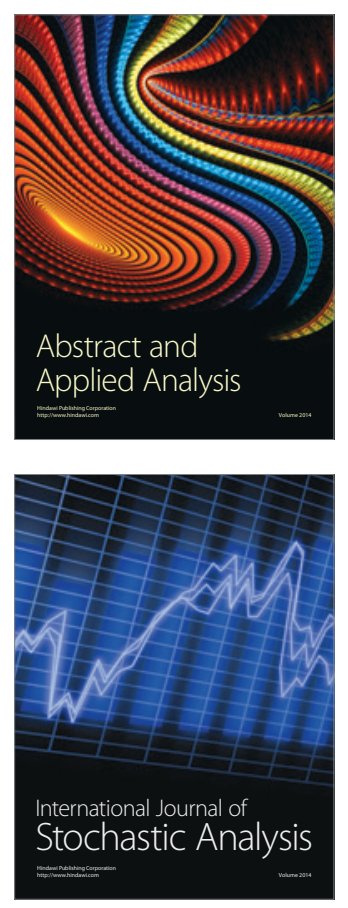

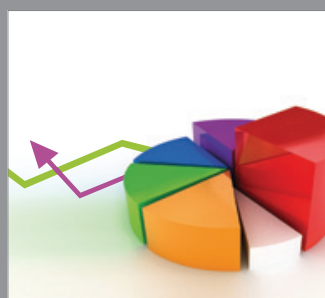

ournal of

Probability and Statistics

Promensencen
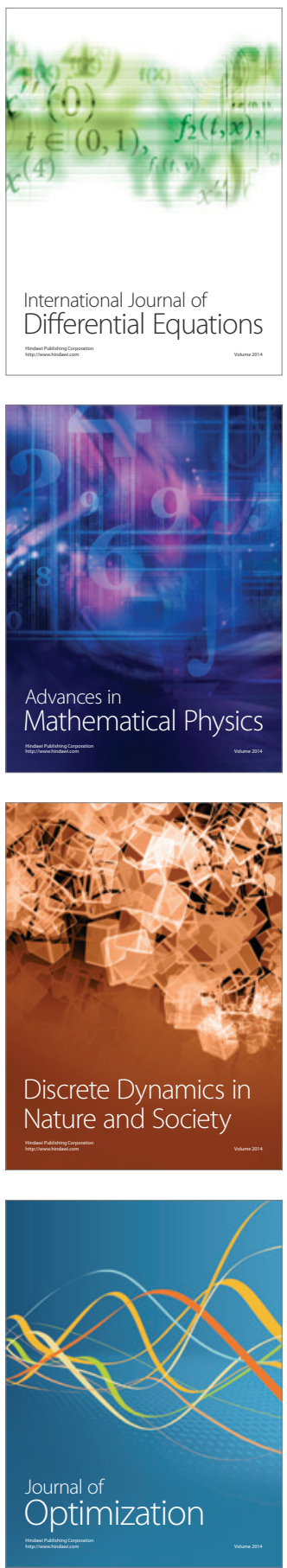